\title{
Real-time image processing for crop/weed discrimination in maize fields
}

\author{
Xavier P. Burgos-Artizzu ${ }^{a}, *$, Angela Ribeiro ${ }^{a}$, Maria Guijarro ${ }^{b}$, Gonzalo Pajares ${ }^{b}$ \\ ${ }^{a}$ Artificial Perception Group (GPA), Centro de Automâtica y Robôtica UPM-CSIC, Spanish National Research Council, Madrid, Spain \\ ${ }^{\mathrm{b}}$ Departamento de Ingeniería del Software e Inteligencia Artificial, Universidad Complutense de Madrid (UCM), Spain
}

Keywords:

Computer vision

Precision Agriculture

Weed detection

Real-time image processing

\begin{abstract}
A B S T R A C T
This paper presents a computer vision system that successfully discriminates between weed patches and crop rows under uncontrolled lighting in real-time. The system consists of two independent subsystems, a fast image processing delivering results in real-time (Fast Image Processing, FIP), and a slower and more accurate processing (Robust Crop Row Detection, $R C R D$ ) that is used to correct the first subsystem's mistakes. This combination produces a system that achieves very good results under a wide variety of conditions. Tested on several maize videos taken of different fields and during different years, the system successfully detects an average of $95 \%$ of weeds and $80 \%$ of crops under different illumination, soil humidity and weed/crop growth conditions. Moreover, the system has been shown to produce acceptable results even under very difficult conditions, such as in the presence of dramatic sowing errors or abrupt camera movements. The computer vision system has been developed for integration into a treatment system because the ideal setup for any weed sprayer system would include a tool that could provide information on the weeds and crops present at each point in real-time, while the tractor mounting the spraying bar is moving.
\end{abstract}

\section{Introduction}

For some years now, governments, researchers and farmers have become increasingly conscious of the important role Precision Agriculture (PA) will play in the near future. With the current projections of expected world population growth and the subsequent decrease of available land and natural resources, there will be a pressing need for a cheaper, more efficient and environmentally friendly agriculture (Srinivasan, 2006). PA seeks to avoid applying the same management practices to a crop regardless of site conditions and may be used to improve field management from several perspectives; for example, it can help to minimize the wastage of pesticides required for the effective control of weeds, diseases and pests and to ensure that crops receive adequate nutrients, leading to more efficient and greener agriculture (Kropff et al., 1997; Earl et al., 1996).

Site-specific weed management practices using chemical tools propose to apply herbicide in the dosage strictly necessary based on both weed infestation and location or position.

Research work in this area is difficult to classify and compare due to the variations among different crop and weed species and to the different approaches taken to collect field data (Thorp and Tian, 2004). However, almost all existing weed detection methods pro- cess the image in two steps: 1 ) segmentation of vegetation against the background (soil and/or harvest residues) and 2) detection of the vegetation pixels that represent weeds.

The procedures for the segmentation of vegetation usually assume that all pixels belonging to vegetation can be easily extracted by some combination of the color planes on the RGB model (Woebbecke et al., 1995; Andreasen et al., 1997; Pérez et al., 2000; Aitkenhead et al., 2003; Yang et al., 2003; Ribeiro et al., 2005; Van Evert et al., 2006). Other approaches propose the use of the HSI color model combined with classification methods such as Bayes networks and clustering (Lee et al., 1996, 1999; Hemming and Rath, 2001; Blasco et al., 2002; Zheng et al., 2009). Segmentation can also be performed by selecting texture features based on their similarities with previous models encountered, stored in a database (Martí et al., 2001; Bosch et al., 2007). Moreover, segmentation can be performed by combining different cameras, such as conventional and NIR cameras (Gerhards and Christensen, 2003). An in-depth review of vegetation segmentation methods can be found in Meyer and Neto (2008).

Once vegetation pixels have been identified, weed detection by computer vision methods is usually performed by combining information on differences in color, position, shape, texture, size or spectrum of weeds and crop. The use of only one or many of these characteristics depends on the way the photographic images are taken, the crop type, and the weed species involved.

Some works present statistical studies of the features involved (Van Evert et al., 2006; Pérez et al., 2000; Andreasen et al., 1997). Others choose to distinguish between species by their different 
spectra, combining the information obtained from a conventional camera with a NIR camera, (Gerhards and Christensen, 2003; Gerhards and Oebel, 2006). In lettuce, plants and weeds can be clearly distinguished by differences in their sizes and position, Blasco et al. (2002). This is similar to the case of cauliflowers, where weeds can be located by their position and some shape characteristics (Onyango and Marchant, 2003), or in carrots and cabbages, where weeds have distinct differences in color and size (Hemming and Rath, 2001).

Other studies make use of classification or feature extraction methods, including as many characteristics as necessary. This is used, for example, in Bayes networks (Lee et al., 1996, 1999; Granitto et al., 2005), or neuronal networks (Aitkenhead et al., 2003; Astrand and Baerveldt, 2002; Burks et al., 2005; Yang et al., 2002; Vioix et al., 2002). Additionally, some studies process the images in the frequency domain (Tian et al., 1999; Tang et al., 1999; Vioix et al., 2002) or even using Fuzzy Logic Yang et al. (2003).

In winter crops, the most common weeds (Avena sterilis and Lolium rigidum) are very difficult to distinguish from crops at early phenological stages due to similarities in their shape, texture and color. The only discriminatory characteristic left is the spatial position occupied by each plant. Moreover, in those cases where the weed has characteristics that distinguish it from the crop (for example, maize crops and weeds such as Sorghum, Cyperus, Xanthium and Datura), sometimes the image processing used to discriminate between them is computationally very expensive, so that discrimination in real time is not profitable. However, the problem becomes real-time approachable if weeds can be detected by their position.

Formally, to detect weeds by position, the vegetation cover growing between two crop rows is considered to be weed cover (Hague et al., 2006; Ribeiro et al., 2005; Tellaeche et al., 2008a; Burgos-Artizzu et al., 2010). Therefore, the initial problem is converted into another one: detecting crop rows and labeling as weeds the vegetation growing in between.

Crop row location in real time is often an important goal in the autonomous guidance of agricultural vehicles (Billingsley and Schoenfisch, 1997; Keihcer and Seufert, 2000; Torii, 2000; Reid et al., 2000; Hague and Tillet, 2001; Gottschalk et al., 2008). However, in this context, the crop rows are roughly approximated by lines, while for weed patch detection the precision required is much higher. Some other works use the Hough transform (Gonzalez and Woods, 2003), to fully locate the crop rows and then label the rest of vegetation pixels as weeds (Leemans and Destain, 2006; Tellaeche et al., 2008b; Gée et al., 2008; Bakker et al., 2008). The drawback of this approach is the high computational complexity of the Hough transform, which makes it unsuitable for applications in which there is a need to process images in real-time, i.e. at $25 \mathrm{fps}$ (frames per second), the standard video camera frame rate. Finally, some other studies deal with simpler images, taken closer to the ground and in such a way that perspective is eliminated, so that crop rows can be more easily located and the processing adapted to real-time (Olsen, 1995; Tillet and Hague, 1999; Hague and Tillet, 2001; Sogaard and Olsen, 2003; Ribeiro et al., 2005).

Unfortunately, none of these studies thus far have resulted in the commercialization of the technologies developed. The major obstacles to commercialization concern the high computing and economic costs involved, as well as the difficulties of correctly representing all of the possible situations present in real and outdoor conditions, (Stafford, 2000; Slaughter and Downey, 2008).

In this context, the ideal setup would be to have at our disposal a tool providing real-time information on weed infestation and location at each point, which could then be used to activate a sprayer in response. This work presents an approach aimed at precisely extracting weed, crop and soil covers from each video frame, in real-time. The main challenge in terms of image analysis is to achieve an appropriate discrimination among weeds, crops and soil

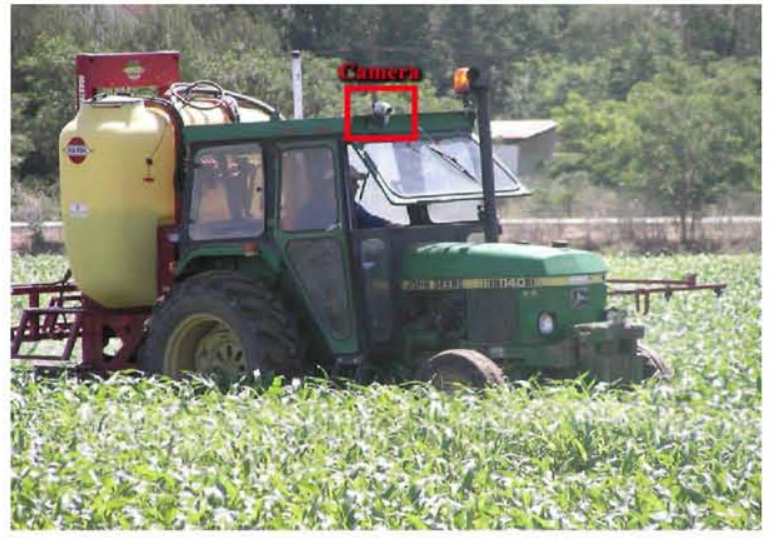

Fig. 1. The camera is placed directly on the roof of the tractor, at a height of $2.15 \mathrm{~m}$ from the ground, with a $10^{\circ}$ pitch angle.

under varying conditions of lighting, soil background texture, crop damage and weed infestation, within the strong processing time limitations imposed by the spraying operation.

The rest of this paper is structured as follows. Section 2 describes the proposed real-time image processing. Section 3 discusses the results obtained. Section 4 outlines the conclusions and future work.

\section{Real-time image processing}

\subsection{Camera placement and video acquisition}

The main goal of this work is to develop a system able to estimate weed seedlings and crop covers from video frames in real-time. These covers can then be used to decide the required herbicide dosage at each point (video frame) (Burgos-Artizzu et al., 2007), and its direct application in real-time can be achieved, for example, through the automation of a commercial spraying bar.

The authors have recently automated a commercial spraying bar, model Hardi NK, of $10 \mathrm{~m}$ of length, distributed in five independent sections of $2 \mathrm{~m}$ each (Burgos-Artizzu, 2009). To use the output of the present computer vision system to control this spraying bar, the images need to cover the $10 \mathrm{~m}$ width of the bar. Therefore, the camera is placed on the uppermost part of the tractor (the roof), at a height of $2.15 \mathrm{~m}$ from the ground, with a pitch angle of $10^{\circ}$, as is shown in Fig. 1.

Using this setup, however, the resulting images span only $6.5 \mathrm{~m}$ of the field instead of the desired $10 \mathrm{~m}$. Unfortunately, placing the camera higher would have other disadvantages, such as much lower precision or higher image blurriness, due to the major impact of tractor jolting. A possible solution could be to place a second camera on the other side of the roof and combine information from two cameras to cover the $10 \mathrm{~m}$. In either case, the image processing method used would be the same as the one presented in this paper. Two different conventional digital video cameras were alternatively tested, Sony DCR PC110E and JVC GR-DV700E. They both capture clear quality images with a resolution of $720 \times 576$ pixels.

The videos were taken in 3 different maize fields in Madrid (Spain), on different days over the last 4 years, during the times of post-emergence herbicide applications (May). Field experiments were conducted in La Poveda Research Farm (Arganda, Central Spain). The climate of the site is Mediterranean Continental with cold winters, hot summers and limited precipitation of about $400 \mathrm{~mm}$. The fields used in this study follow the normal agricultural practices in this culture. Fields had an average of 3.0 ha and were planted in early April with $0.75 \mathrm{~m}$ row spacing and a population of 90,000 plants/ha. Fields were sprinkler irrigated, with the 


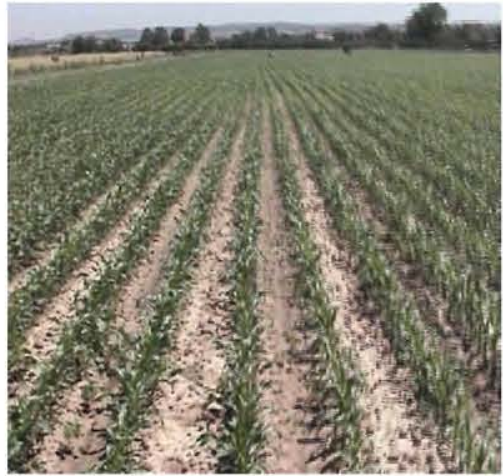

a

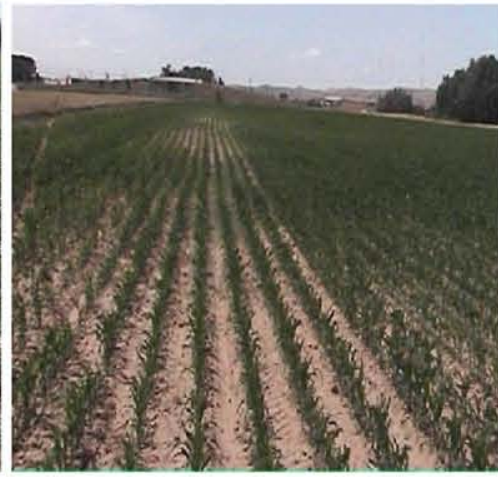

b

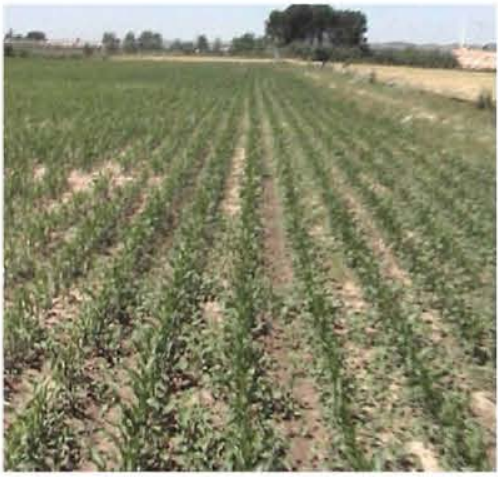

C

Fig. 2. Image frame examples.

first irrigation applied 1 month after planting and weekly irrigations thereafter. Weeds were assessed in May when maize was at the stage $14-16$ of BBCH scale, Donald (2006). The fields have been rotated with winter barley, and showed a normal infestation of weeds with some patches of S. Halepense, X. Strumarium, Datura Stramonium $L$. and $D$. Ferox, with leaf sizes varying from $6 \mathrm{~cm}$ to $75 \mathrm{~cm}$. Herbicide treatments, fertilization and other agricultural operations were also those commonly used in maize crops. The tractor speed was kept constant and was set around $7 \mathrm{~km} / \mathrm{h}$, the standard speed for weed spraying.

Sampling was purposedly carried out with different weather and field conditions to have a video footage representing a broad variety of real conditions. Actual video database consists of more than $16 \mathrm{~h}$ of footage, from sunny and cloudy days with varying levels of air humidity, and showing different weed/crop growth stage (within previously stated limits). Fig. 2 shows an example of the type of image frames obtained. As can be seen from the figure, the frames captured can vary greatly in illumination, soil background and crop/weed coverage, all of which are uncontrollable outdoor situations to which the image processing should be robust. Moreover, the image processing will have to deal with the issues raised by the displacement of the tractor through an irregular terrain, which can cause blurry images and even sudden displacement of the angle of view. Finally and most importantly, the image processing has to do all of this under great time limitations because the processing speed should be at least 25 fps considering that vehicle's treatment speed must be about $2 \mathrm{~m} / \mathrm{s}(7 \mathrm{~km} / \mathrm{h})$.

\subsection{Real-time image processing}

Real-time image processing at $25 \mathrm{fps}$ means that each frame has to be processed in $0.04 \mathrm{~s}$. However, due to the difficulty of the task at hand, a simple exploration of the image will not be sufficient to extract all of the crop and weed pixels precisely. Therefore, the proposed image processing method is divided into two different steps. The first step (Robust Crop Row Detection, $R C R D$ ) includes all of the operations necessary to detect the crop rows correctly under all circumstances, regardless of the processing time needed. That information is then passed to the second step (Fast Image Processing, FIP), which adapts its results to the current frame. RCRD is considered a fail-safe against possible changes that may occur in the middle of a run, like dramatic changes in vegetation conditions or any of the problems produced by the jolting of the tractor and the irregularities of the terrain (displacements, changes on the viewing angle, image blurriness).

Fig. 3 shows the image processing flow diagram for each new frame. Both steps of the processing method start working simultaneously with the segmented binary image, where vegetation pixels are separated from non-vegetation ones. Because RCRD does not have to work in real-time, several frames will be captured while it is still working on previous data. All those frames are stored, and as soon as $R C R D$ is ready to process new data, it retrieves all of them, combines them and performs its operations to detect crop rows, storing the final result in the Crop Rows reference image. Meanwhile, FIP processes each new frame independently, using the last Crop Rows image created by RCRD. In the following sections, each part is discussed separately.

\subsubsection{Vegetation segmentation}

The first step, which is common to almost all image processing weed detection methods, is the segmentation of the vegetation pixels. To discriminate vegetation pixels, a linear combination of the RGB planes with coefficients $(r=-0.884, g=1.262, b=-0.311)$ was performed. These coefficients were found using a genetic algorithm optimization, and proved to perform better than Excess Green coefficients ( $r=-1, g=2, b=-1$ ) (Woebbecke et al., 1995), on similar images, Burgos-Artizzu et al. (2010). Then the threshold used for binarization is set automatically for each image. The automatic adjustment of the threshold value is crucial to achieving segmentation robust to changes in illumination, which occur frequently due to changing weather, especially in party cloudy days.

Two different threshold adjusting methods were evaluated: 1 ) Otsu's widely used Otsu (1979) method and 2) setting the threshold to the mean pixel intensity, following Eq. (1). Fig. 4 shows the result of both methods on two different input frames. Otsu's method tends to set the threshold value higher than the mean pixel intensity, causing vegetation to be slightly exaggerated. Moreover, Otsu's method is slower, so the mean intensity method was chosen instead. This choice is further validated by previous work, Burgos-Artizzu et al. (2010), where threshold values returned by a genetic algorithm optimization were much closer to the average of intensities than Otsu's, both in maize and winter cereal images.

Threshold $=\frac{\sum_{x=1}^{N} \sum_{y=1}^{M}(r * R(x, y)+g * G(x, y)+b * B(x, y))}{M * N}$

where, $r=-0.884, g=1.262, b=-0.311$.

\subsubsection{Robust Crop Row Detection (RCRD)}

$R C R D$ detects the crop rows pixels, creating a reference image, Crop Rows, which will then be used by FIP. This reference image is essential not only to guarantee the precision of the results but also to prevent all sorts of errors.

Because RCRD is time-independent, several frames will be captured while $R C R D$ is still busy processing previous frames. These 


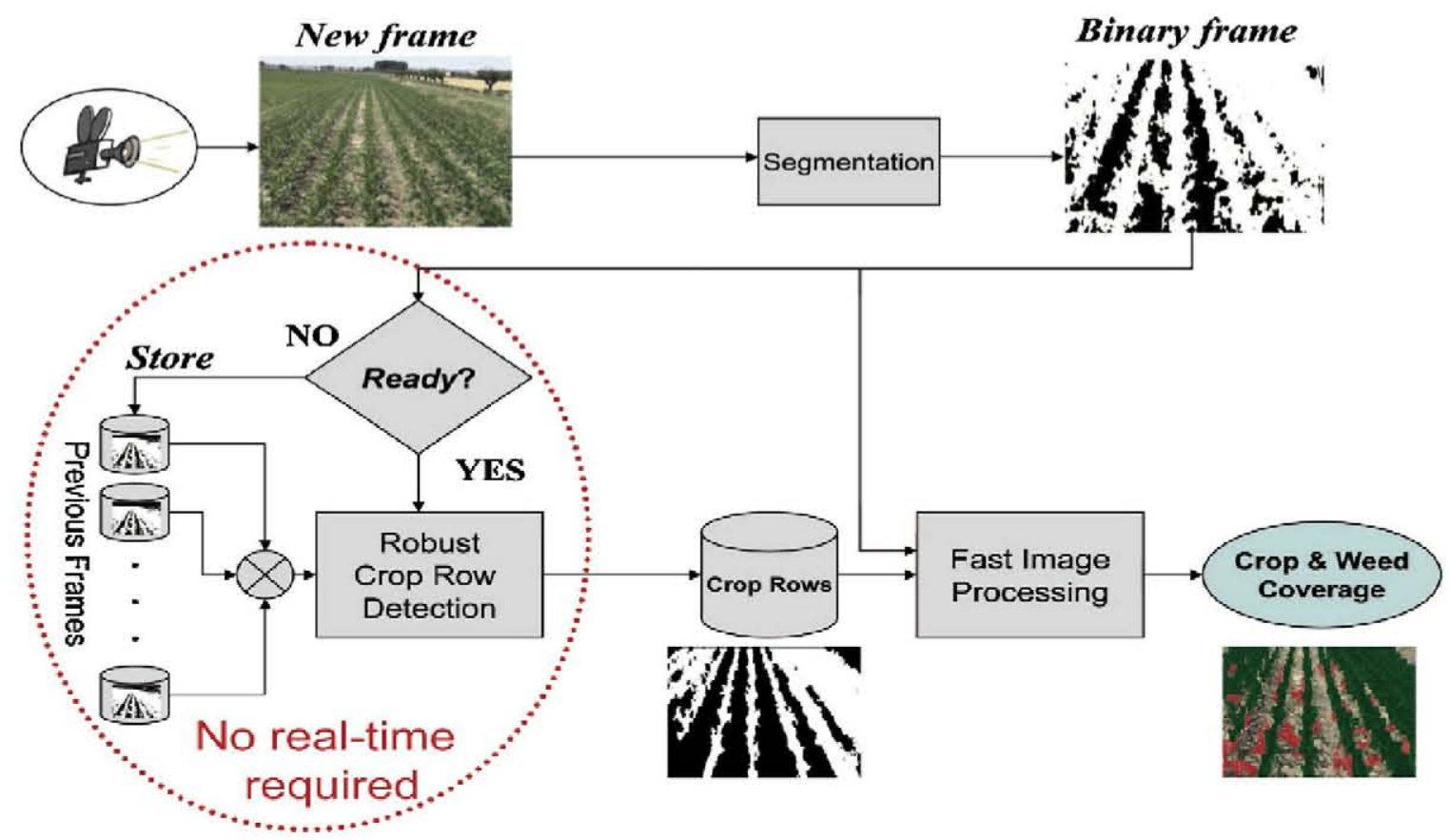

Fig. 3. Flow diagram of the proposed image processing method.

frames are stored, waiting for $R C R D$ to be ready for a new processing. Then the first step of RCRD is to combine all of the stored binary frames into a single image with an AND operation. The AND operation yields an image where only the persistent vegetation pixels are kept. Vegetation pixels that persist over a set of consecutive frames, given the movement of the tractor, will mostly be crop rows pixels, except for some weed pixels if a large weed patch is encountered. Fig. 5 shows an example, where 8 consecutive frames are combined.
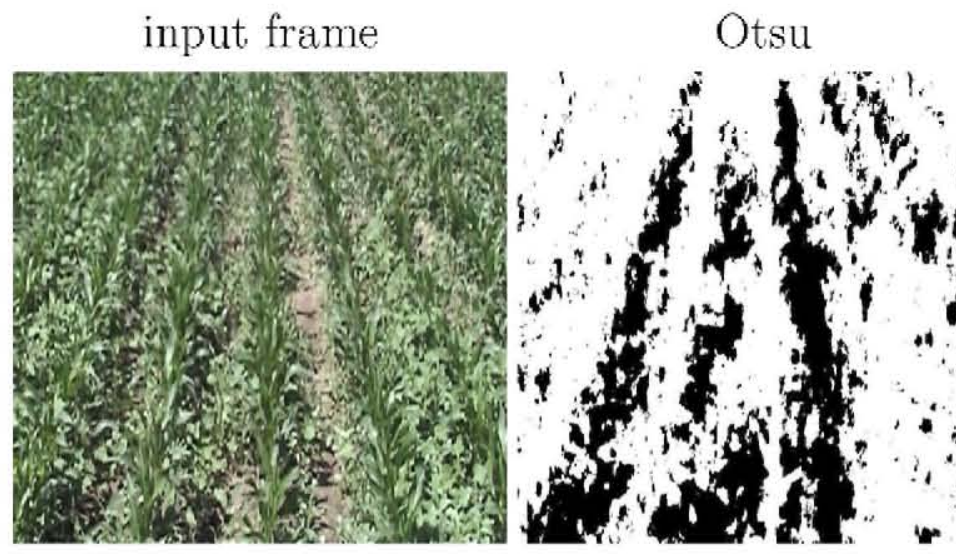

\section{Mean intensity}
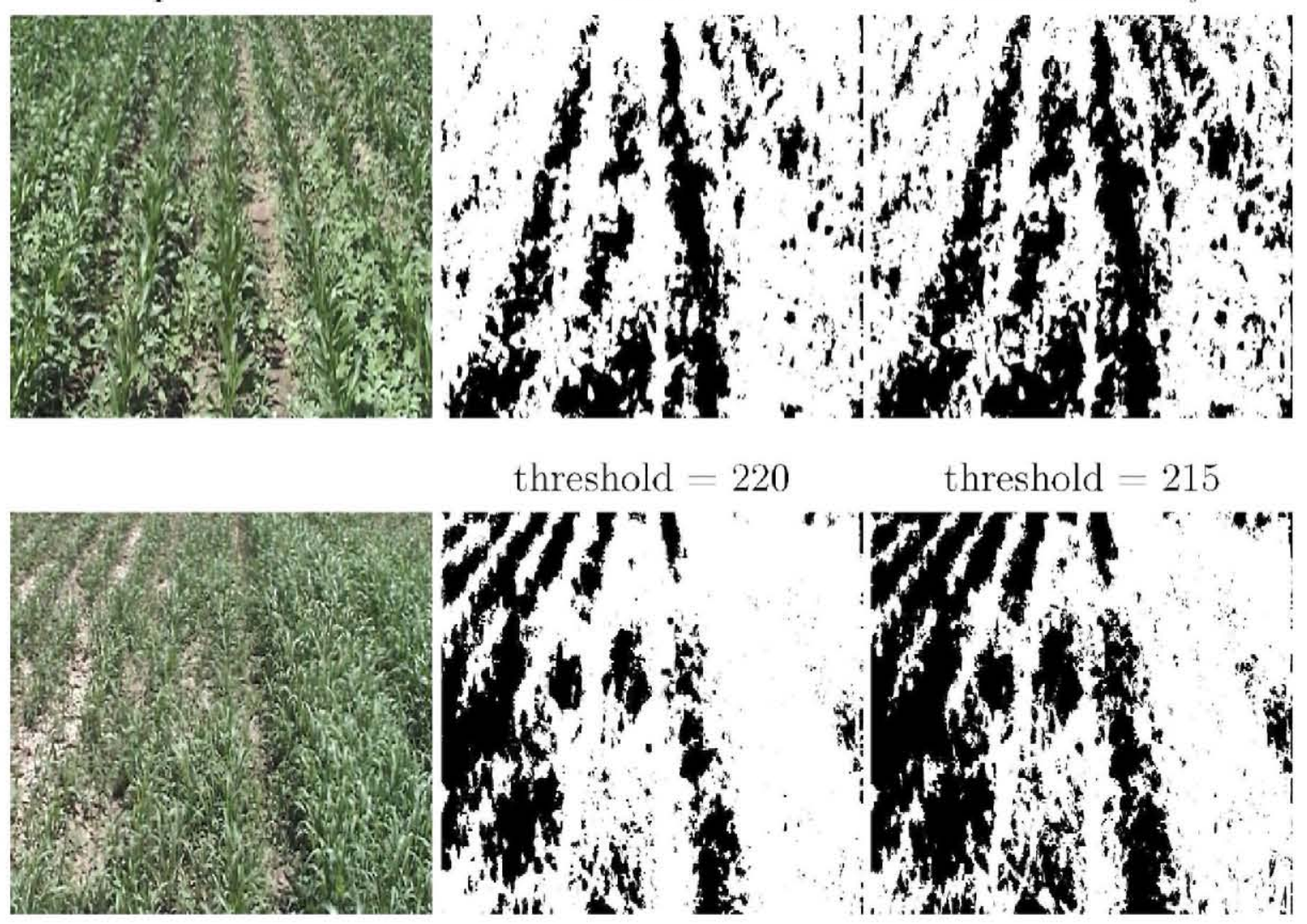

threshold $=231$

threshold $=221$

Fig. 4. Comparison of the two automatic threshold value adjustment methods, Otsu's and mean intensity, tested on two different input frames. 


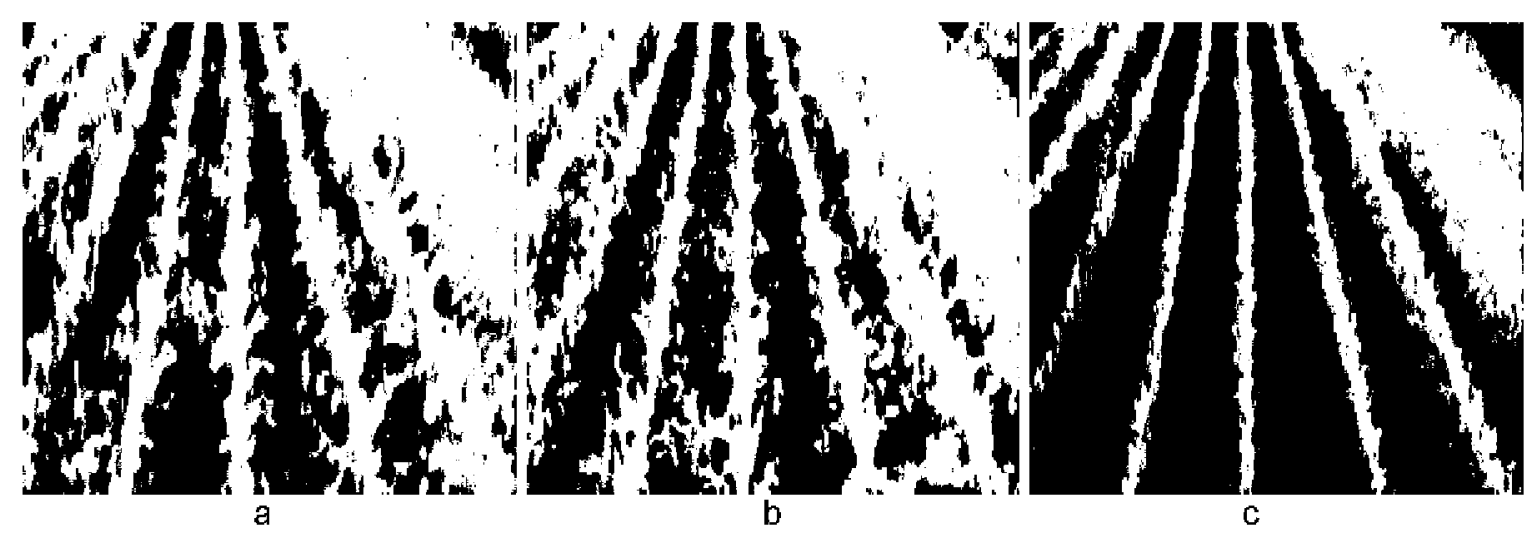

Fig. 5. AND operation on 8 consecutive frames to select crop rows. (a) First frame; (b) eighth and last frame; (c) AND result on all 8 consecutive frames.

However, the AND operation alone will not be enough if large weed patches are present in the images, as can be seen in Fig. 6(a) and (b). To cope with these cases, RCRD performs a morphological opening of the image followed by region extraction and filtering all those regions too small to be considered a full crop row. The morphological opening is used to clean the image, Fig. 6(c), prior to the region extraction. Then crop rows are easily recog- nizable by their much larger areas than the rest of the regions (typically 7-10 times bigger than the remaining weed regions), Fig. 6(d).

Fig. 7 shows three examples of the final result of RCRD, where crop rows are successfully detected. $R C R D$ needs an average of $0.3 \mathrm{~s}$ per processing, which at $25 \mathrm{fps}$ means that it will run once every eight frames.

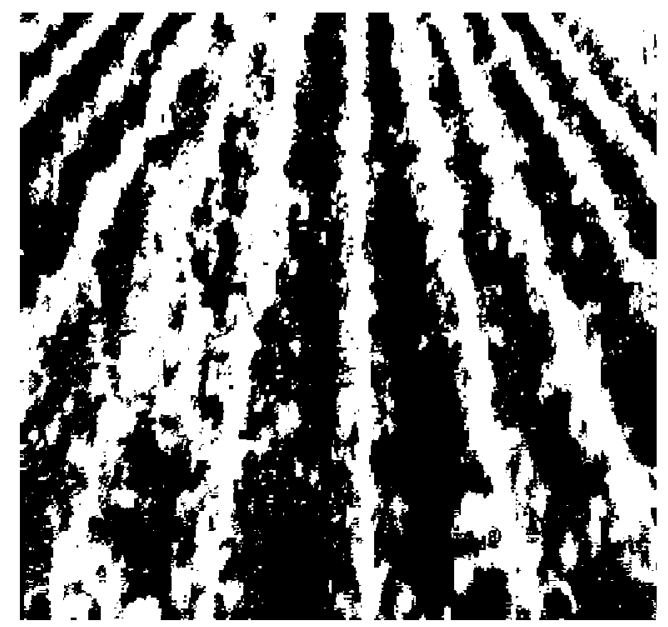

a

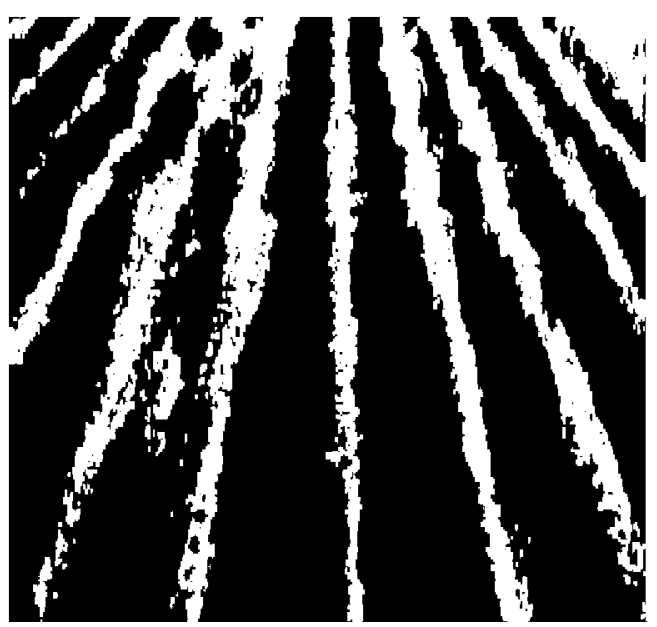

C

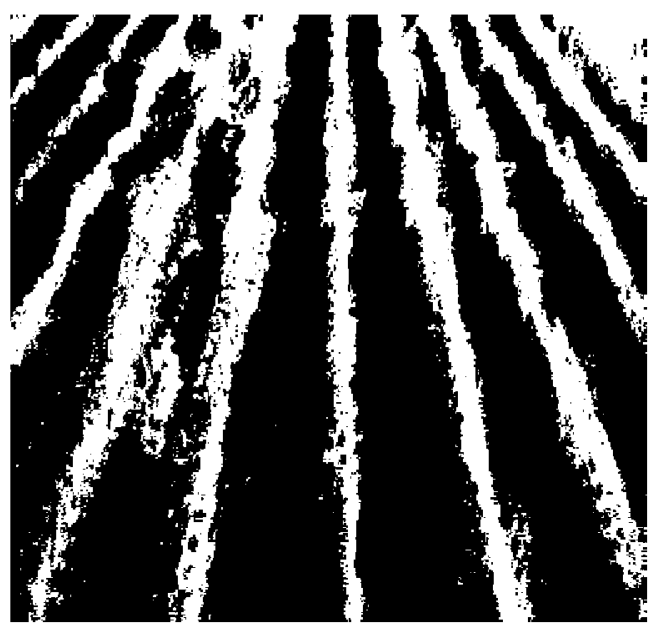

b

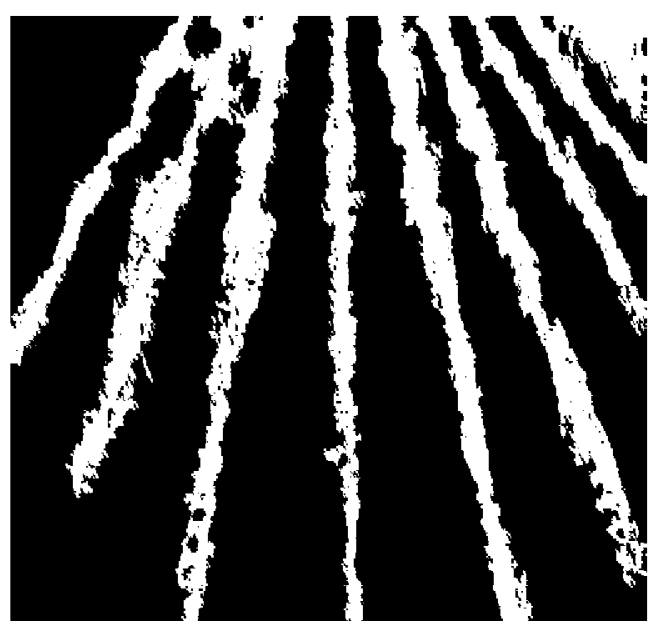

d

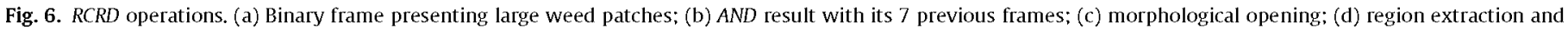
filtering by size, where regions with areas less than 2000 pixels are eliminated. 

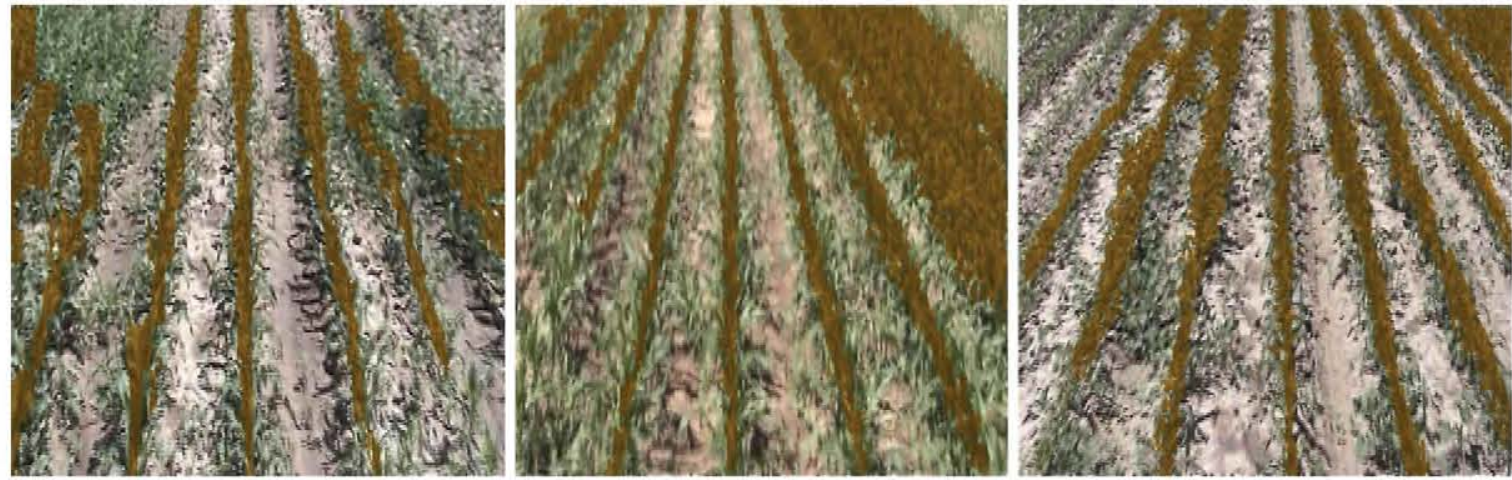

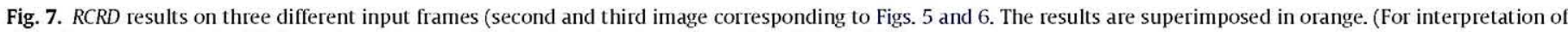
the references to color in this figure legend, the reader is referred to the web version of the article.)

\subsubsection{Fast Image Processing (FIP)}

FIP is hugely limited by the response time needed for a real-time image processing at $25 \mathrm{fps}$. The image is divided into horizontal strips, and for each strip, a vector with as many components as the number of columns in the binary image is created and filled, at each component, with the number of white pixels in the corresponding column (inside the current horizontal strip), Eq. (2).

$V(x)=\sum_{x=\text { strip_tart }}^{\text {strip_end }} \operatorname{Image}(x, y)$

Then, the vector is explored searching for several consecutive high values, and marking those positions as potentially being part of the crop rows. A value is considered high if it is higher than $3 / 4$ of the height of the strip (number of rows). Several consecutive high values are considered to be a sequence of more than a given threshold number of consecutive high values. This threshold is calibrated initially using the minimum value of consecutive high values present in the bottom strip of the Crop Rows image created by RCRD. This threshold is, roughly, the expected minimum width of the crop rows at the bottom of the image. All steps in the procedure are illustrated in Fig. 8, using as a threshold 5\% of the image width (36 pixels in this case).

To take into account the effect of perspective, which slightly reduces crop rows width in upper parts of the image an average of $2.5 \%$ with current setup, the threshold is reduced for each strip by a factor of $\left(0.025 / n_{\text {_strips }}\right)$. The size (height) of each strip, however, remains fixed throughout the image. The number of strips used can vary, but for the purpose of this paper it was fixed to 25 (16 pixels/strip). This is the best setup found on trial and error on the videos processed for Section 3.

The result of this procedure, dividing the image into 25 strips, can be seen in Fig. 9(a). As expected, all crop rows are clearly marked. However, medium and large sized weed patches are also marked. To distinguish between the crop rows and weeds, FIP uses the Crop Rows image created by RCRD. All sets of positions marked by FIP that coincide with at least one crop row pixel of the Crop Rows image will be kept, while all other sets of positions will be discarded. This can be seen in Fig. 9(b) and (c). Fig. 9(b) shows the union of the Crop Rows image (in blue) with the pixels marked by FIP (green). Fig. 9(c) shows the discarded sets (in red).

Finally, once the crop rows are extracted, weed pixels can be determined simply as all other pixels that were part of the vegetation after segmentation and are not considered crop. Fig. 10 shows the final result of the whole system on 4 different input images (crops in green, weeds in red) that represent a variety of situations. The proposed methods were implemented in the $\mathrm{C}++$ programming language and compiled as DirectShow filters, to enable their use in Windows platforms directly, using pre-installed applications like GraphEdit. All of the video capture and processing was performed using a mid-range laptop computer (Intel dual core T2400 CPU, $1.83 \mathrm{GHz}, 1 \mathrm{~Gb}$ Ram).
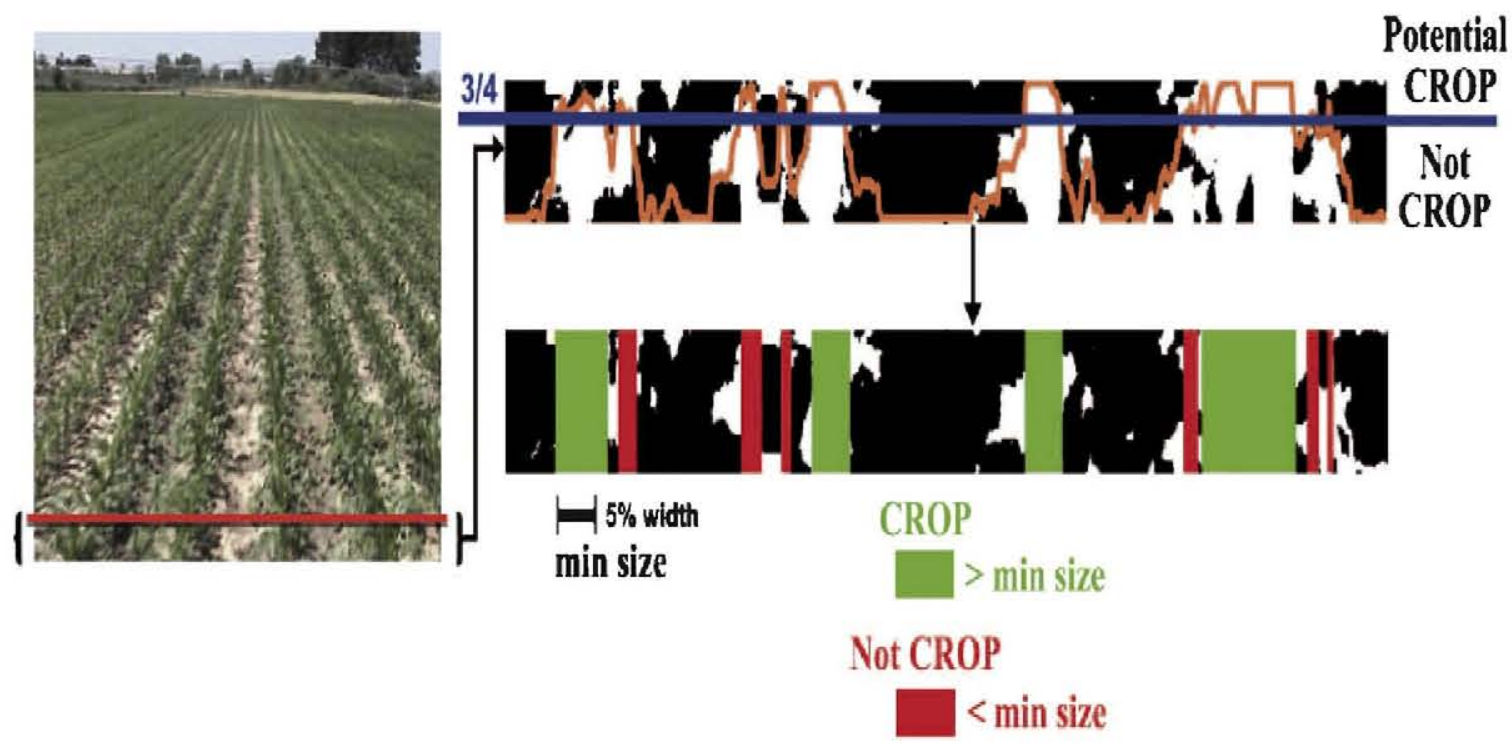

Fig. 8. Fast Image Processing operations. 


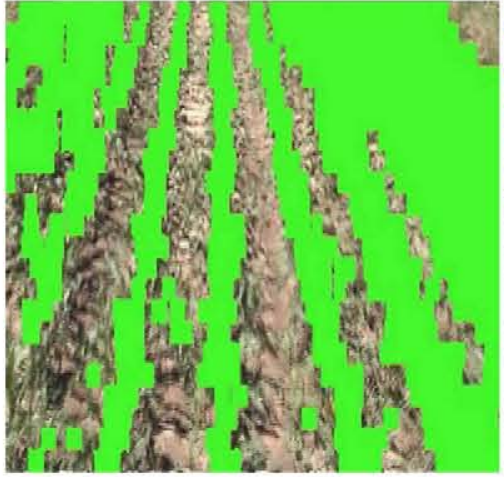

a

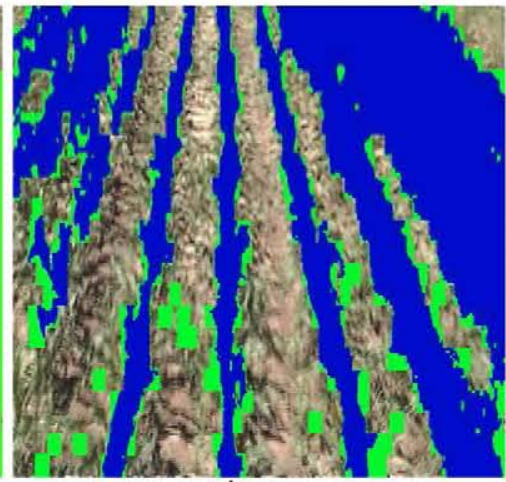

b

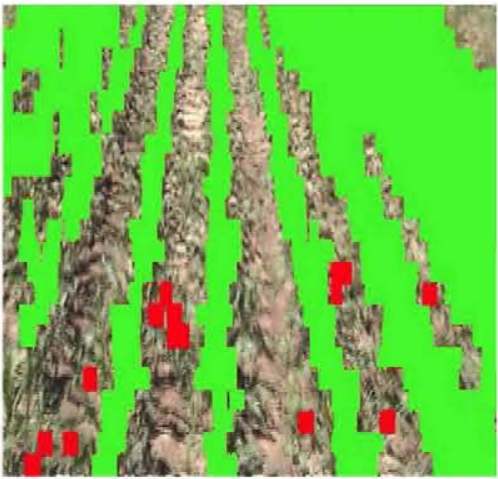

C

Fig. 9. Image Processing results. (a) FIP results (green). (b) Mixed FIP (green) and RCRD results (blue). (c) Correction of FIP results using the Crop Rows image created by RCRD (discarded sets in red). (For interpretation of the references to color in this figure legend, the reader is referred to the web version of the article.)

\section{Results and discussion}

Six video segments from different years and fields, thus representing different situations, were chosen and processed using the system, recording the results obtained for each frame. Then the same frames were processed manually, labeling soil, crop and weed pixels using image editing software. The automatic and manual identifications were then compared to evaluate the system's results. Each one of the 6 videos has an average $12 \mathrm{~s}$ duration (300 frames), having therefore a total of 1800 frames. Due to the dif- ficulty of manual labeling, of those 1800 frames, only 300 were manually labeled ( 50 per set, randomly chosen).

Fig. 11 shows the result of the system on some illustrative frame examples, while Table 1 shows the full system results over all six video sets. The first three sets (Fair1, Fair2 and Fair3) contain the most common situations. A situation is considered fair when crop rows are clearly visible (there are no evident sowing errors) and weeds have a maximum horizontal size of $60 \mathrm{~cm}$ (they don't fill the whole space between two consecutive crop rows). An example of this standard situation can be seen in Fig. 11(a). Three different fair
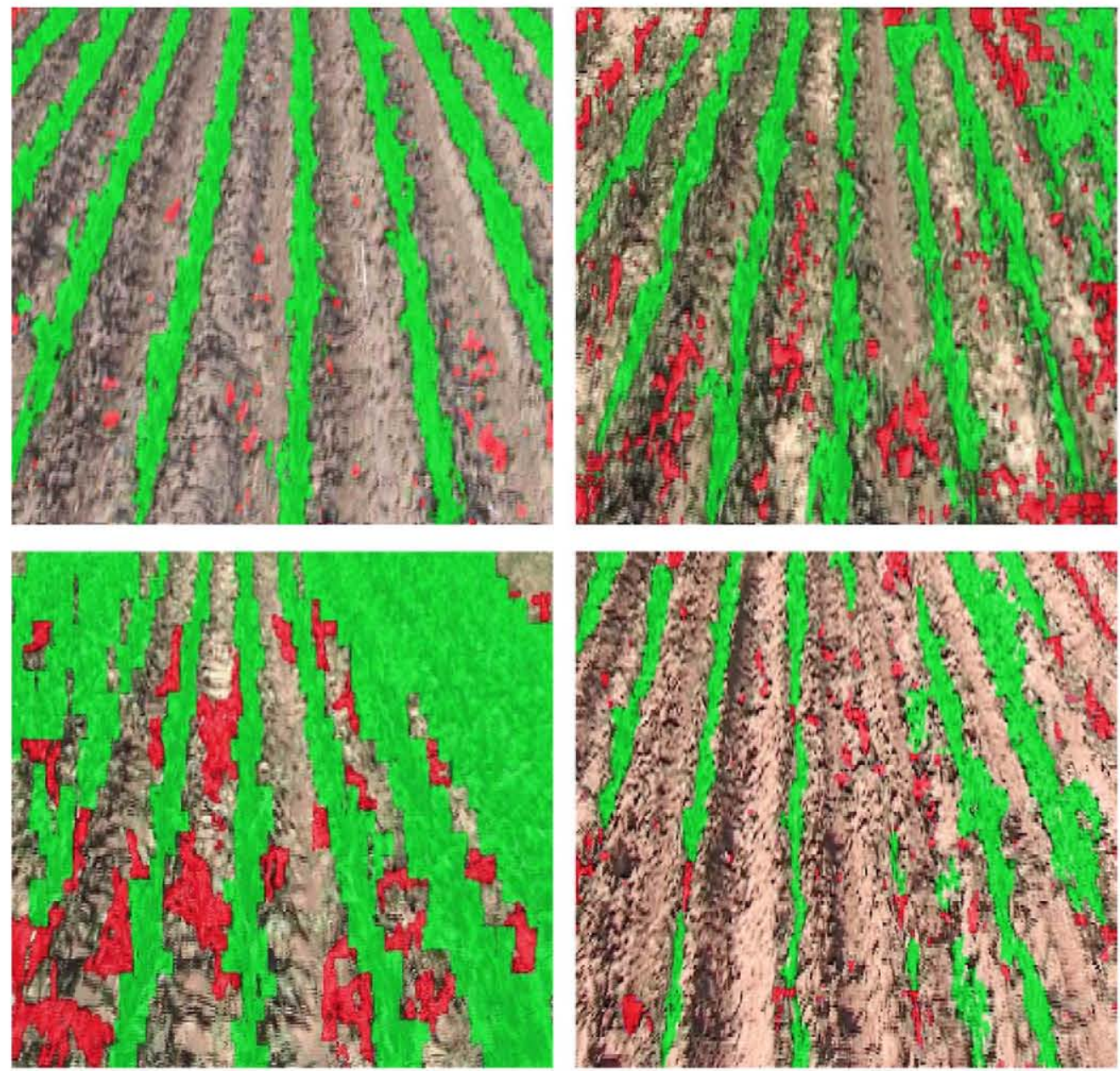

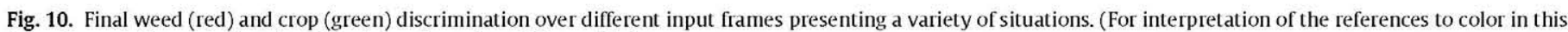
figure legend, the reader is referred to the web version of the article.) 


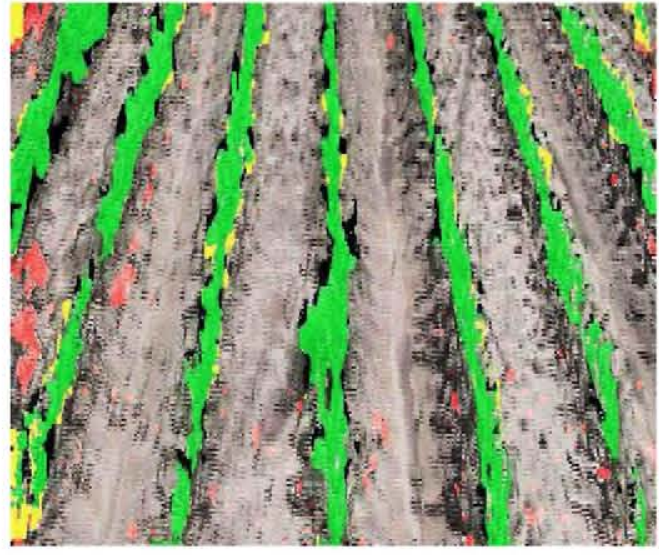

a Fair1

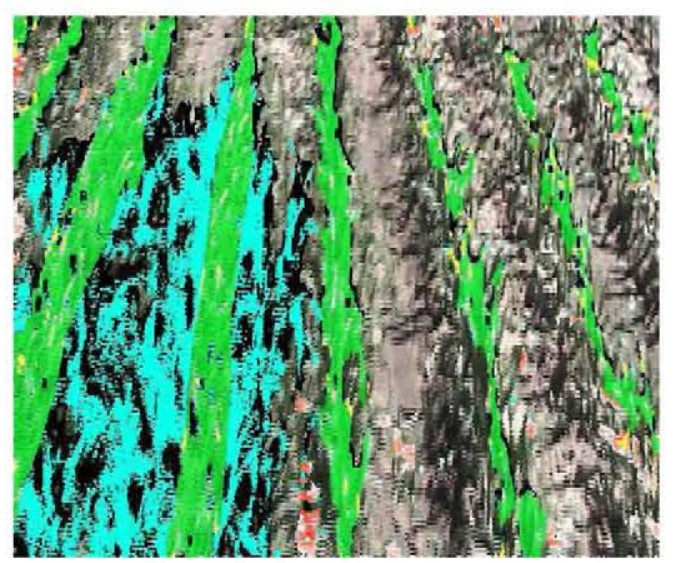

c Patches

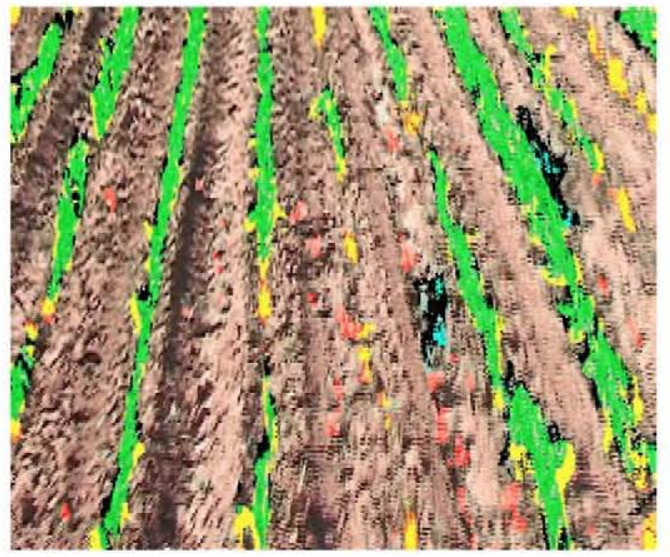

b Sowing Errors

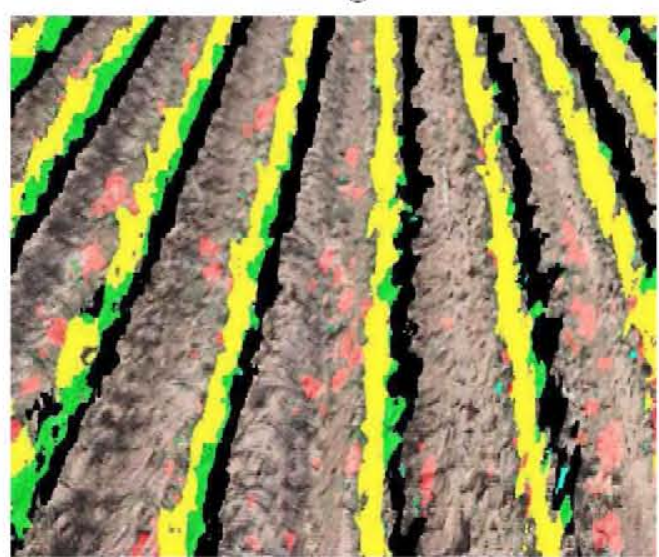

d Movement

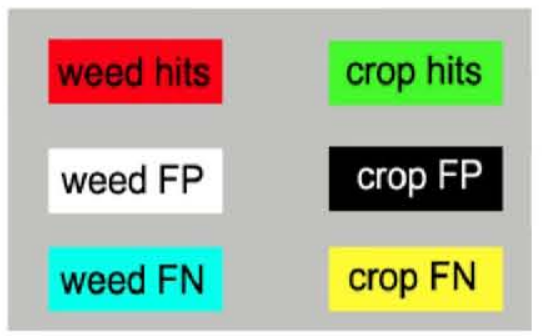

Fig. 11. System results over each video set.

sets were chosen to represent a wide variety of illumination, soil humidity and weed/crop growth conditions that can be encountered. The other three sets (Sowing Errors, Patches and Movement) contain more difficult and uncommon situations, such as the presence of sowing errors, Fig. 11(b), very large weed patches, Fig. 11(c), or sequences with abrupt camera movements due to significant terrain irregularities, Fig. 11(d).

The results are analyzed in terms of the mean percentage of crop and weed pixels correctly labeled (true positive or hits), false positives and false negatives. It is important to analyze both weed

Table 1

Results of the system.

\begin{tabular}{|c|c|c|c|c|c|c|}
\hline & \multicolumn{3}{|l|}{ Weed } & \multicolumn{3}{|l|}{ Crop } \\
\hline & Hits (\%) & False positives (\%) & False negatives (\%) & Hits (\%) & False positives (\%) & False negatives (\%) \\
\hline Fair1 & 97.3 & 1.7 & 0.9 & 77.6 & 10.3 & 12 \\
\hline Fair2 & 93.4 & 5.4 & 1.2 & 83.2 & 6.3 & 9.5 \\
\hline Fair3 & 95.2 & 4.1 & 0.7 & 81.7 & 7 & 11.3 \\
\hline Sowing errors & 64.9 & 32.4 & 2.6 & 65.9 & 8.2 & 25.8 \\
\hline Patches & 65 & 4.8 & 31.2 & 70 & 27.3 & 2.7 \\
\hline Movement & 74.8 & 13.8 & 11.2 & 36.3 & 31.3 & 32.3 \\
\hline Average ${ }^{*}$ & 85.1 & 11.4 & 3.3 & 68.9 & 12.6 & 18.2 \\
\hline
\end{tabular}

* Average is computed without taking into account Patches set. 
and crop detection because the crop density provides a measure of the weed infestation risk (Ribeiro et al., 2005). Additionally, weed false positives are preferable to weed false negatives because weed patches are less likely to be missed and uncontrolled.

As can be seen both in Table 1 and Fig. 11(a), in fair conditions the proposed image processing method yields very satisfactory results. The system is robust to varying illumination conditions, as well as to constant small changes in the viewing angle and/or image blurriness due to tractor jolting and small terrain irregularities. It achieves on average $95 \%$ and $80 \%$ weed and crop hits, respectively, keeping at all times a very low ratio of weed false negatives (around $1 \%$ ), the most undesirable error, and weed false positives $(3.7 \%$ on average). As expected, crops are more difficult to adjust at a pixel level, due to the fact that FIP does not process every single crop pixel in the image separately. This results in higher levels of both crop false positives and negatives, $7.5 \%$ and $11 \%$ in average, respectively. Because the conditions labeled as fair are encountered over $90 \%$ of the time in the video database, this results fully validate the image processing. However, more difficult and uncommon situations were also analyzed because they still represent real situations that can be encountered.

When the system encounters dramatic sowing errors, as in Fig. 11(b), the system fails to recognize part of the crop due to its discontinuity, mistaking it for weeds, causing high weed false positives (32\%) and crop false negatives (25\%). If very large weed patches are encountered, as in Fig. 11(c), the issue is the opposite: the weed patch is mistaken for crop, causing high crop false positives (27\%) and weed false negatives (31\%). Finally, if during the run there is a very abrupt change in the direction of motion, as in Fig. 11(d), the synchronization between the last RCRD result and FIP each 8 frames is not fast enough, causing an erroneous crop detection (31\% false positives, $32 \%$ false negatives) with subsequent high weed false positives (13\%) and false negatives (11\%).

The weed false positives produced during sowing errors are acceptable errors, considering the difficulty presented in the images, where crop and weeds are difficult to distinguish even for human experts. The weed false negatives produced with large weed patches, however, are a more worrisome issue. To overcome it, images in which there is a high proportion of vegetation pixels after segmentation can be labeled directly as $100 \%$ weeds because they represent situations where herbicide will have to be sprayed at maximum dosage. For example, a threshold of $60 \%$ can be used (Fig. 11(c) has a $64 \%$ of vegetation pixels).

The issues raised due to the movement of the tractor can only be overcome through video stabilization, either via hardware or software. However, most of the tractor movement is staggered in such a way that the synchronization between FIP and RCRD each 8 frames is fast enough. It is only very rarely that such an abrupt displacement as the one observed in the Movement set is encountered, so the cost of implementing video stabilization would not be justified in a first approach, although it should be studied in detail in a future study.

The average results of the system on all sets but Patches, which will always be labeled as $100 \%$ weeds, are very satisfactory, reaching $85 \%$ of weed hits with only $3 \%$ of weed false negatives, and $69 \%$ of crop hits.

\section{Conclusions and future work}

To achieve site-specific management of weeds, the first and most important step is the location and density estimation of weeds. In this context, the development of real-time computer vision based weed detection methods can be of great help in constructing fully automatic and cheap weed sampling systems. The problem does not have a trivial solution, due to changing condi- tions of illumination, humidity, vegetation growth, different weed species encountered, and due to the similarities presented, in many cases, by weeds and crops, making discrimination a complex task and an open field of research.

In this paper, a real-time computer vision-based weed detection system is presented. The system is divided into two different and independent steps to achieve greater precision and robustness to all possible situations encountered, while still being able to produce a real-time outcome. The first step, RCRD, performs all of the operations necessary (no matter how much time is needed) to extract the crop rows present in the image correctly, creating the Crop Rows reference image. The Crop Rows image is then used to correct the results of the real-time step, FIP, which due to its time limitations, is not able to perform a correct discrimination under all circumstances on its own.

The combination of a fast processing system delivering results in real-time $(F I P)$ with a more exhaustive and slower processing system $(R C R D)$, results in a system that achieves very good results in real-time over a wide variety of conditions. Tested on several maize videos taken on different fields and during different years, the system successfully detects an average of $85 \%$ of weeds and $69 \%$ of crops under different illumination, soil humidity and camera displacement/blurriness conditions, even when presented with very difficult weed/crop growth states, such as when the crop rows are not visible due to sowing errors. When presented with fair images, that is small to medium sized weeds and clearly visible crop rows, which represent over $90 \%$ of the situations encountered in the recorded videos, the system successfully detects an average of $95 \%$ of weeds and $80 \%$ of crops, while maintaining a very low rate of weed false negatives (1\%) at all times.

The segmentation method proposed is the key to the robustness of the system to changing illumination conditions. The color indices used $(r=-0.884, g=1.262, b=-0.311)$ create a gray image that can then be easily binarized using a very simple and fast automatic threshold adjustment method (average intensity value), resulting in a very simple and robust segmentation method.

These results were obtained using a conventional video camera placed on the roof of the tractor, and the video capture and processing is performed using a standard laptop PC, so that the system is easy to set up and very inexpensive, two important requisites for its commercialization and use by farmers.

In the near future, the system will also be tested directly on real fields, feeding its output to the fuzzy controller developed by the group (Burgos-Artizzu et al., 2007) which determines the optimum herbicide dosage using expert knowledge and controls the spraying bar in real-time accordingly.

\section{Acknowledgments}

The Spanish Ministry of Education and Science (MEC) and the European Union have provided full and continuing support for this research work through projects PLAN NACIONAL-AGL2008-04670C03-02/AGR (PERICIA) and UE-CP-IP245986-2(RHEA).

\section{References}

Aitkenhead, M., Dalgetty, I., Mullins, C., McDonald, A., Strachan, N., 2003. Weed and crop discrimination using image analysis and artificial intelligence methods. Comput. Electron. Agr. 39, 157-171.

Andreasen, C., Rudemo, M., Sevestre, S., 1997. Assessment of weed density at an early stage by use of image processing. Weed Res. 37, 5-18.

Ast rand, B., Baerveldt, A., 2002. An agricultural mobile robot with vision-based per ception for mechanical weed control. Auton. Robot. 13, 21-35.

Bakker, T., Wouters, H., Van Asselt, K., Bontsema, J., Tang, L., Muller, I., Van Straten, G., 2008. A vision based row detection system for sugar beet. Comput. Electron. Agr. 60, 87-95.

Billingsley, J., Schoenfisch, M., 1997. The successful development of a vision guidance system for agriculture. Comput. Electron. Agric. 16, 147-163. 
Blasco, J., Aleixos, N., Roger, J., Rabatel, G., Moltó, E., 2002. Robotic weed control using machine vision. Biosyst. Eng. 83 (2), 149-157.

Bosch, A., Muñoz, X., Freixenet, J., 2007. Segmentation and description of natural outdoor scenes. Image Vision Comput. 25, 727-740.

Burgos-Artizzu, X., 2009. Percepción y Actuación para el tratamiento selectivo de malas hierbas en tiempo real. Universidad Complutense de Madrid, Tesis Doctoral.

Burgos-Artizzu, X.P., Ribeiro, A., de Santos, M., 2007. Controlador borroso multivariable para el ajuste de tratamientos en agricultura de precisión. Revista Iberoamericana de Automática e Informática Industrial 4 (April (2)), 64-71.

Burgos-Artizzu, X.P., Ribeiro, A., Tellaeche, A., Pajares, G., Fernández-Quintanilla, C., 2010. Analysis of natural images processing for the extraction of agricultural elements. Image Vision Comput. 28, 138-149.

Burks, T., Shearer, S., Heath, J., Donouhue, K., 2005. Evaluation of neural network classifiers for weed species discrimination. Biosyst. Eng. 91 (3), 293-304.

Donald, W.W., 2006. Estimated corn yields using either weed cover or rated control after pre-emergence herbicides. Weed Sci. 54 (2), 373-379.

Earl, R., Wheeler, P., Blackmore, B., Godwin, R., 1996. Precision farming: the management of variability. Landwards $51(4), 18-23$.

Gée, C., Bossu, J., Jones, G., Truchetet, F., 2008. Crop/weed discrimination in perspective agronomic images. Comput. Electron. Agr. 60, 49-59.

Gerhards, R., Christensen, S., 2003. Real-time weed detection, decision making and patch spraying in maize, sugarbeet, winter wheat and winter barley. Weed Res. $43,385-392$.

Gerhards, R., Oebel, H., 2006. Practical experiences with a system for site-specific weed cont rol in arable crops using real-time image analysis and gps-cont rolled patch spraying. Weed Res. 46, 185-193.

Gonzalez, R.C., Woods, R.E., 2003. Digital Image Processing, 2nd ed. Prentice-Hall.

Gottschalk, R., Burgos-Artizzu, X.P., Ribeiro, A., Pajares, G., Sánchez-Miralles, A. December 2008. Real-time image processing for the guidance of a small agricultural field inspection vehicle. In: 15 th International Conference on Mechat ronics and Machine Vision in Practice (M2VIP'08), Auckland, New Zealand.

Granitto, P., Verdes, P., Ceccatto, H., 2005. Large-scale investigation of weed seed identification by machine vision. Comput. Electron. Agr. 47, 15-24.

Hague, T., Tillet, N., 2001. A bandpass filter-based approach to crop row locartion and tracking. Mechatronics 11, 1-12.

Hague, T., Tillet, N., Wheeler, H., 2006. Automated crop and weed monitoring in widely spaced cereals. Precis. Agr. 7, 21-32.

Hemming, J., Rath, T., 2001. Computer-vision based weed identification under field contions using cont rolled lighting. J. Agr. Eng. Res. 78 (3), 233-243.

Keihcer, R., Seufert, H., 2000. Automatic guidance for agricultural vehicles in Europe. Comput. Electron. Agr. 25, 169-194.

Kropff, M., Wallinga, J., Lotz, L., 1997. Modelling for precision weed management. In: Precision Agriculture: Spatial and Temporal Variability of Environmental Quality. Wiley, Chester, pp. 182-204.

Lee, W., Slaughter, D., Giles, D., 1996. Development of a machine vision system for weed control using precision chemical application. In: Proceedings of International Conference on Agricultural Machinery Engineering ‘96, Seoul, Korea, pp. $802-811$.

Lee, W., Slaughter, D., Giles, D., 1999. Robotic weed control system for tomatoes using machine vision and precision chemical application. Precis. Agr. 1, $95-113$.

Leemans, V., Destain, M.-F., 2006. Line cluster detection using a variant of the hough transform for culture row localisation. Image Vision Comput. 24, 541-550.

Martí, J., Freixenet, J., Batlle, J., Casals, A., 2001. A new approach to outdoor scene description based on learning and topdown segmentation. Image Vision Comput. $19,1041-1055$.
Meyer, G., Neto, J., 2008. Verification of color vegetation indices for automated crop imaging applications. Comput. Electron. Agr. 63, 282-293.

Olsen, H., 1995. Determination of row position in small-grain crops by analisis of video images. Comput. Electron. Agr. 12, 147-162.

Onyango, C., Marchant, J., 2003. Segmentation of row crop plants from weeds using colour and morphology. Comput. Electron. Agr. 39, 141-155.

Otsu, N., 1979. A threshold selection method from gray-level histograms. IEEE Trans. Syst. Man Cybern. 9, 62-66.

Pérez, A., López, F., Benlloch, J., Christensen, S., 2000. Colour and shape analysis techniques for weed detection in cereal fields. Comput. Electron. Agr. 25, 197-212.

Reid, J., Zhang, Q., Noguchi, N., Dickson, M., 2000. Agricultural automatic guidance research in north America. Comput. Electron. Agr. 25, 155-167.

Ribeiro, A., Fernández-Quintanilla, C., Barroso, J., García-Alegre, M.C., 2005. Development of an image analysis system for estimation of weed. In: Proceedings 5 th European Conf. On Precision Agriculture (5ECPA), pp. 169-174.

Slaughter, D., D.K., G., Downey, D., 2008. Autonomous robotic weed control systems: a review. Comput. Electron. Agr. 61, 63-78.

Sogaard, H., Olsen, H., 2003. Determination of crop rows by image analysis without segmentation. Comput. Electron. Agr. 38, 141-158.

Srinivasan, A., 2006. Handbook of Precision Agriculture. Principles and Applications. The Haworth Press, New York.

Stafford, J.V., 2000. Implementing precision agriculture in the 21st century. J. Agr. Eng. Res. 76, 267-275.

Tang, L., Tian, L, Steward, B., Reid, J., 1999. Texture-based weed classification using gabor wavelets and neural network for real-time selective herbicide applications. Trans. ASAE 99, 7035.

Tellaeche, A., Burgos-Artizzu, X.P., Pajares, G., Ribeiro, A., 2008a. A new vision-based approach to diffe rential spraying in precision agriculture. Comput. Electron. Agr. $60,144-155$

Tellaeche, A., Burgos-Artizzu, X.P., Pajares, G., Ribeiro, A., 2008b. A vision-based method for weeds identification through the bayesian decision theory. Pattern Recognit. 41, 521-530.

Thorp, K, Tian, L, 2004. A review on remote sensing of weeds in agriculture. Precis. Agr. 5, 477-508.

Tian, L., Reid, J., Hummel, J., 1999. Development of a precision sprayer for site-specific weed management. Trans. ASAE 42, 893-900.

Tillet, N., Hague, T., 1999. Computer-vision based hoe guidance for cereals an initial trail. J. Agr. Eng. Res. 74, 225-326.

Torii, T., 2000. Research in autonomous agriculture vehicles in Japan. Comput. Electron. Agr. 25, 133-153.

Van Evert, F.K., Van Der Heijden, G., Lotz, L., Polder, G., Lamaker, A., De Jong, A. Kuyper, M., Groendijk, E, Neeteson, J., Van Der Zalm, T., 2006. A mobile field robot with vision-based detection of volunteer potato plants in a corn crop. Weed Technol. 20, 853-861.

Vioix, J., Douzals, J., Truchetet, F., Assêmat, L., Guillemin, J., 2002. Spatial and spectral methods for weed detection and localization. EURASIP JASP 7, 679-685.

Woebbecke, D., Meyer, G., VonBargen, K., Mortensen, D., 1995. Color indices for weed identification under various soil, residue, and lighting conditions. Trans. ASAE $38(1), 271-281$.

Yang, C., Prasher, S., Landry, J., pp. 7.15-7.22 2002. Weed recognition in corn fields using back-propagation neural network models. In: Canadian Byosistems Engineering, vol. 44.

Yang, C, Prasher, S., Landry, J., H.S., R., 2003. Development of an image processing system and a fuzzy algorithm for site-specific herbicide applications. Precis. Agr. $4,5-18$.

Zheng, L., Zhang, J., Wang, Q, 2009. Mean-shift-based color segmentation of images containing green vegetation. Comput. Elect ron. Agr. 65, 93-98. 